\title{
Corrigendum
}

\section{Delayed postprandial TAG peak after intake of SFA compared with PUFA in subjects with and without familial hypercholesterolaemia: a randomised controlled trial}

Linn K. L. Øyri, Patrik Hansson, Martin P. Bogsrud, Ingunn Narverud, Geir Florholmen, Lena Leder, Marte G. Byfuglien, Marit B. Veierød, Stine M. Ulven and Kirsten B. Holven

(First published online [15 May 2018])

doi: https://doi.org/10.1017/S0007114518000673

In Figure 2, the denomination of the lipoprotein subclasses (XXL, XL, L, M, S and XS VLDL, IDL, L, M and S LDL, XL, L, M and S HDL) should read $\mathrm{mol} / \mathrm{l}$ and not $\mathrm{mmol} / \mathrm{l}$. The figure has been corrected and resupplied below.
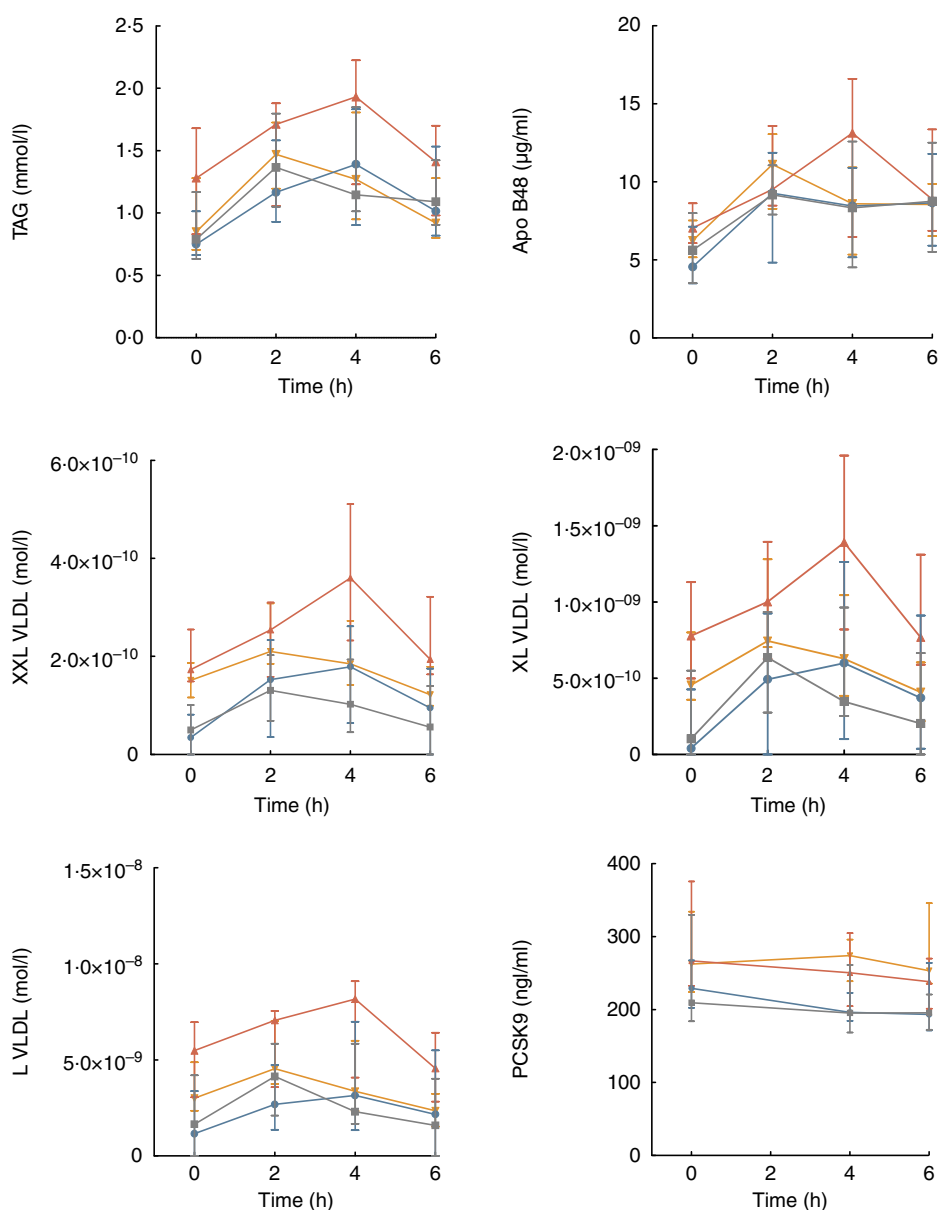

Fig. 2. Postprandial response in TAG, apoB48, the three largest VLDL sub-classes and proprotein convertase subtilisin/kexin type 9 . Values are medians and interquartile ranges. $P$ values from analysis of total and incremental AUC (AUC and IAUC) in a linear mixed model for repeated measures. PCSK9, proprotein convertase subtilisin/kexin type 9; FH, familial hypercholesterolaemia; $P_{\text {meal }}, P$ value SFA v. PUFA meal; $P$ group, $P$ value FH v. controls. - , FH, SFA; - , FH, PUFA; - , controls, SFA; - , controls, PUFA. 